\title{
Efecto de la inclusión de aceite esencial de orégano en la dieta de lechones destetados sobre parámetros productivos
}

\author{
Effect of the inclusion of oregano essential oil in the diet of weaned piglets on \\ productive parameters
}

\author{
Nelson Baca C. ${ }^{1}$, Antonio Ampuero B. ${ }^{1,2}$
}

\section{Resumen}

\begin{abstract}
El estudio tuvo como objetivo evaluar el efecto de la suplementación de aceite esencial de orégano sobre los parámetros productivos en el posdestete de lechones. El trabajo se realizó en una granja comercial porcina en Ventanilla, Callao, Perú. La suplementación fue por 12 días. Se utilizaron 120 lechones distribuidos de manera aleatoria en 8 corrales de 15 animales. Los tratamientos fueron: T1, control, con una dieta de preinicio con $18 \%$ de proteína, 600 ppm de amoxicilina y 55 ppm de carbadox; T2, dieta como en T1 más suplementación con 500 ppm de aceite esencial de orégano (Origanum vulgare). Se evaluó la ganancia de peso, consumo de alimento e índice de conversión alimenticia. Se halló un menor consumo de alimento en T2 $(1.58 \mathrm{~kg})$ respecto a T1 $(1.84 \mathrm{~kg})(\mathrm{p}<0.05)$. La ganancia de peso y el índice de conversión alimenticia no se vieron afectados por los tratamientos.
\end{abstract}

Palabras clave: lechones; posdestete; aceite esencial de orégano; parámetros productivos

\section{Abstract}

The aim of this study was to evaluate the effect of the supplementation of essential oil of oregano on the productive parameters in weaned piglets. The study was carried out in a commercial pig farm in Ventanilla, Callao, Peru. The supplementation was for 12 days. One hundred and twenty piglets randomly allocated in 8 pens of 15 animals were distributed

\footnotetext{
${ }^{1}$ Laboratorio de Zootecnia y Producción Agropecuaria, Facultad de Medicina Veterinaria, Universidad Nacional Mayor de San Marcos, Lima, Perú

${ }^{2}$ E-mail: aampuerob@unmsm.edu.pe

Recibido: 3 de agosto de 2018

Aceptado para publicación: 12 de octubre de 2019
} 
in two treatments: $\mathrm{T} 1$, control, with a pre-start diet with $18 \%$ protein, $600 \mathrm{ppm}$ amoxicillin and 55 ppm carbadox; T2, diet as in T1 plus supplementation with 500 ppm of oregano essential oil (Origanum vulgare). The weight gain, feed consumption and feed conversion ratio were evaluated. A lower feed intake was found in T2 $(1.58 \mathrm{~kg})$ compared to T1 (1.84 $\mathrm{kg})(\mathrm{p}<0.05)$. The weight gain and the feed conversion index were not affected by the treatments.

Key words: piglets; post-weaning; oregano essential oil; productive parameters

\section{INTRODUCCIÓN}

La industria porcina en el Perú ha tenido un incremento en los últimos años. El consumo per cápita en Lima en 2001 era aproximadamente de $2.5 \mathrm{~kg}$ y en el resto del país de $3.5 \mathrm{~kg}$. Actualmente, el consumo per cápita de cerdo en Lima alcanza los $6 \mathrm{~kg} /$ año y en el resto del país es de $4.5 \mathrm{~kg} / \mathrm{año}$ (MINAGRI, 2013). Por otro lado, el Ministerio de Agricultura y Riego tiene como meta incrementar el consumo de carne de cerdo en el país, con diferentes eventos que buscan dar a conocer las bondades de la carne de cerdo con el objetivo de llegar a consumir $10 \mathrm{~kg}$ per cápita por año de esta carne en la próxima década (Minagri, 2018).

El aumento en la demanda de carne de cerdo impulsa a la industria a mejorar la productividad en las granjas. Se requiere cerdos con mayor velocidad de crecimiento, mayor rendimiento de carcasa y mayor prolificidad. Una de las acciones a tomar sería reducir el tiempo de la lactación de la marrana; es decir, destetar precozmente al lechón para lograr más partos por año (Paramio et al., 2012).

El destete en el lechón produce cambios en la morfología intestinal y en el $\mathrm{pH}$ y, como consecuencia, modificación de la microbiota intestinal. Así mismo, pasan por un periodo de estrés debido al cambio de corral, separación de la madre y agrupamiento con otros lechones. Por tanto, hay disminu- ción del consumo voluntario de alimento y problemas digestivos por la deficiencia en la producción enzimática (Castillo y Alves, 2007). Las prácticas que se realizan en las granjas para lograr mitigar los efectos negativos del destete precoz se enfocan en utilizar dietas semi-húmedas, dietas con alto contenido de lactosa, así como el uso de acidificantes, antibióticos promotores de crecimiento (APC), probióticos, prebióticos y extractos vegetales (Danura, 2010).

Actualmente, la utilización de plantas y hierbas medicinales o de alguno de sus componentes se vislumbra como una de las alternativas más naturales. Algunas plantas contienen aceites esenciales que les confieren propiedades aromáticas. Diferentes experimentos han demostrado que el uso de estos aceites puede producir un aumento de la ganancia diaria de peso similar a lo registrado con APC en cerdos y aves (García et al., 2007; Guerra et al., 2008; Ayala et al., 2011).

No se conoce en su totalidad el mecanismo de acción de estas sustancias, pero se considera que disminuyen la oxidación de los aminoácidos, ejercen una acción antimicrobiana sobre algunos microorganismos intestinales, favorecen la absorción intestinal, aumentan la secreción de enzimas digestivas, aumentan la palatabilidad de los alimentos y estimulan su ingestión, o mejoran el estado inmunológico del animal (Piva y Rossi, 1999). La Comisión Europea ha registrado los aceites esenciales y sus compuestos como saborizantes en la alimentación. Los 
saborizantes tales como el carvacrol, timol, eugenol, limoneno, mentol, cinamaldehido, carvona y $\mathrm{p}$-cimeno no presentan riesgos para la salud del consumidor, y el orégano (Origanum vulgare) es una fuente de estos (Burt y Reinders, 2003).

El orégano tiene cualidades antioxidantes y antimicrobianas contra organismos patógenos (Windisch et al., 2008). Se ha postulado que aumenta la tasa de renovación celular de la mucosa intestinal debido a los fenoles naturales que contiene, manteniendo una población más saludable de enterocitos (Bruerton, 2002). Es por esto que el aceite esencial de orégano y sus metabolitos son de particular interés para los productores de alimento y criadores de cerdos debido a la facilidad de administración, beneficios y retorno económico de la inversión (Pearce et al., 2011). Por lo tanto, el objetivo de este estudio fue evaluar el efecto del aceite esencial de orégano sobre algunos parámetros productivos en lechones recién destetados.

\section{Materiales y Métodos}

\section{Lugar del Estudio}

El estudio se realizó en la granja comercial porcina «Granja Huerto San Martín de Porres» situada en el distrito de Ventanilla, Provincia Constitucional del Callao, Lima, Perú. La parte experimental se realizó en abril de 2015.

\section{Animales}

Se seleccionaron 120 lechones con una edad promedio de 24 días, de la línea genética PIC del cruce Camborough (hembras) y MP427 (verraco). Los lechones tuvieron pesos entre 6 y $6.6 \mathrm{~kg}$ y procedieron del área de maternidad de la granja. El alimento suministrado era una preparación comercial (Pig Tech, dieta basal) ofrecida ad libitum. El aceite esencial de orégano fue proporcionado a través del producto comercial Orevito ${ }^{\circledR}-\mathrm{L}$ (CKM, Perú), destinado para aportar $40 \mathrm{~g}$ de carvacrol por litro de producto (CKM, 2010).

\section{Instalaciones}

Los lechones estuvieron alojados en un galpón de 20 × $5 \mathrm{~m}$, dividido en 8 corrales de $5 \mathrm{~m}^{2}$. Los corrales contaban con pisos slats de plástico con un área de $3 \mathrm{~m}^{2}$ por lechón. Cada corral tenía un comedero circular de plástico con tolva de $50 \mathrm{~cm}$ de radio y dos dispensadores de agua con chupones $3 / 8$ y un volumen de agua de $21 / \mathrm{min}$. La calefacción fue provista por briquetas, obteniendo una temperatura promedio de $26{ }^{\circ} \mathrm{C}$.

\section{Diseño del Estudio}

Los 120 lechones estuvieron distribuidos en ocho corrales de 15 cerdos cada uno donde se destinaron a uno de dos tratamientos con cuatro repeticiones por tratamiento: $\mathrm{T}_{1}$ : Dieta basal (Pig Tech 1 medicado con $600 \mathrm{ppm}$ de amoxicilina y $55 \mathrm{ppm}$ de carbadox) y $\mathrm{T}_{2}$ : Dieta basal $+500 \mathrm{ppm}$ de aceite esencial de orégano.

$\mathrm{Al}$ inicio del estudio los lechones fueron pesados en una balanza electrónica Mettler Toledo ${ }^{\circledR}$ BBA231-W con capacidad para 300 $\mathrm{kg}$ y aproximación de $0.05 \mathrm{~kg}$. Para pesar los ingredientes se utilizó una balanza digital con capacidad de $5 \mathrm{~kg}$ y aproximación de 0.02 $\mathrm{kg}$. Para medir el aceite esencial de orégano se utilizó una jeringa de $1 \mathrm{ml}$ y aproximación de $0.01 \mathrm{ml}$. Los parámetros productivos evaluados fueron ganancia de peso $(\mathrm{kg})$, consumo de alimento $(\mathrm{kg})$ y conversión alimenticia.

\section{Análisis Estadístico}

Los datos fueron analizados mediante el programa estadístico SAS v. 9.0. El efecto de la incorporación del aceite esencial de orégano en la dieta sobre la ganancia de peso, consumo de alimento y conversión alimenticia fue comparado mediante un análisis de 
Cuadro 1. Ganancia de peso de lechones destetados alimentados con una dieta comercial (T1) y suplementados con aceite esencial de orégano (T2)

\begin{tabular}{cccc}
\hline Grupo & $\begin{array}{c}\text { Peso Inicial } \\
(\mathrm{kg})\end{array}$ & $\begin{array}{c}\text { Peso final } \\
(\mathrm{kg})\end{array}$ & Ganancia de peso $(\mathrm{kg})$ \\
\hline $\mathrm{T} 1$ & 6.264 & 7.843 & $1.579^{\mathrm{a}}$ \\
$\mathrm{T} 2$ & 6.272 & 7.769 & $1.497^{\mathrm{a}}$ \\
\hline
\end{tabular}

a Superíndices iguales indican que no hay diferencia estadística significativa

Cuadro 2. Consumo de alimento de lechones destetados alimentados con una dieta comercial (T1) y suplementados con un producto conteniendo aceite esencial de orégano (T2)

\begin{tabular}{cccc}
\hline Grupo & $\begin{array}{c}\text { Alimento ofrecido } \\
(\mathrm{kg})\end{array}$ & $\begin{array}{c}\text { Alimento residual } \\
(\mathrm{kg})\end{array}$ & $\begin{array}{c}\text { Consumo } \\
(\mathrm{kg})\end{array}$ \\
\hline $\mathrm{T} 1$ & 2.098 & 0.258 & $1.840^{\mathrm{a}}$ \\
$\mathrm{T} 2$ & 1.805 & 0.228 & $1.612^{\mathrm{b}}$ \\
\hline
\end{tabular}

a,b Superíndices diferentes indican diferencia estadística significativa $(p<0.05)$

varianza para un diseño completamente al azar. Se consideraron significativos los valores de $\mathrm{p}<0.05$.

\section{Resultados y Discusión}

La ganancia de peso de los lechones fue similar entre los dos tratamientos $(\mathrm{p}=0.171)$ (Cuadro 1). En este sentido, Guerra et al. (2008) encontraron una mayor ganancia de peso en lechones suplementados con el aceite esencial de orégano frente a una dieta sin APC, pero menor cuando los lechones fueron suplementados con APC. Asimismo, Shiva (2007) y Parrado et al. (2006) encontraron mayores ganancias de peso en lechones destetados suplementados con aceite esencial de orégano en comparación con aquellos suplementados con APC. En otras especies, Ayala et al. (2011) hallaron una mayor ganancia de peso en conejos que recibieron $1 \%$ de orégano en la dieta versus los conejos que no recibieron esa suplementación. Asimismo, Martínez (2012) halló una mayor ganancia de peso en pollos de carne cuando se adicionaba aceite esencial de orégano a la dieta.

Se observó una diferencia significativa $(p=0.006)$ a favor del grupo control (T1) en términos de consumo de alimento (Cuadro 2). Por otro lado, Guerra et al. (2008) no encontraron diferencia en el consumo de alimento en cerdos recién destetados en un experimento similar, mientras que en aves, Ayala et al. (2006) hallaron que el consumo de alimento mostró una diferencia a favor del grupo control sin suplemento de aceite esencial de orégano. Estos autores sugieren que la presencia de metabolitos secundarios como los flavonoides, derivados fenólicos del tipo de terpenos y sesquiterpenos presentes en el orégano tienen acción irritante sobre las mucosas afectando el consumo de alimento. Así mismo, se ha comprobado que el carvacrol puede presentar respuestas adversas en el consumo de alimento y ganancia de peso en pollos de engorde (Lee et al., 2003). 
Cuadro 3. Ganancia de peso $(\mathrm{kg})$ e índice de conversión alimenticia de lechones destetados alimentados con una dieta comercial (T1) y suplementados con un producto conteniendo aceite esencial de orégano (T2)

\begin{tabular}{lcc}
\hline Parámetro productivo & T1 (Control) & $\begin{array}{c}\text { T2 (aceite esencial de } \\
\text { orégano) }\end{array}$ \\
\hline Ganancia de peso $(\mathrm{kg})^{1}$ & $1.579^{\mathrm{a}}$ & $1.497^{\mathrm{a}}$ \\
Índice de conversión alimenticia & $1.165^{\mathrm{a}}$ & $1.077^{\mathrm{a}}$ \\
\hline
\end{tabular}

$a, b$ Superíndices diferentes dentro de filas indican diferencia estadística significativa $(p<0.05)$

${ }^{1}$ Periodo de 12 días posdestete

En el índice de conversión alimenticia tampoco se encontró diferencia significativa entre tratamientos ( $\mathrm{p}=0.112$; Cuadro 3), concordando con los hallazgos de Guerra et al. (2008) en lechones destetados. Igualmente, Shiva et al. (2012) tampoco encontraron una diferencia en la conversión alimenticia de pollos de carne al comparar dietas suplementadas con aceite esencial de orégano versus APC. Así mismo, Martínez (2012) y Ayala et al. (2011) encontraron que la inclusión de aceite esencial de orégano en la dieta mejora el índice de conversión alimenticia en pollos de carne y conejos, respectivamente. Por otro lado, Lara et al. (2010) utilizando diferentes aceites esenciales en pollos de carne concluyeron que las combinaciones de aceites esenciales disminuyen la conversión de alimento, pero incrementan la viabilidad de la parvada. Según Parrado et al. (2006), el orégano puede mejorar la conversión alimenticia en los lechones al aumentar la digestibilidad de los nutrientes y favorecer el equilibrio de la microflora, disminuyendo el potencial de adhesión de patógenos en el epitelio intestinal.

\section{Conclusión}

La suplementación de 500 ppm de aceite esencial de orégano (Orevitol ${ }^{\circledR}-\mathrm{L}$ ) produjo una respuesta significativa negativa en el consumo de alimento durante los primeros 12 días post-destete de lechones, sin causar diferencias significativas en la ganancia de peso ni en el índice de conversión alimenticia.

\section{Literatura Citada}

1. Ayala L, Martínez M, Acosta A, Dieppa O, Hernández L. 2006. Una nota acerca del efecto del orégano como aditivo en el comportamiento productivo de pollos de ceba. Cuban J Agr Sci 40: 455-458.

2. Ayala L, Silvana N, Zocarrato I, Gómez S. 2011. Utilización del orégano vulgar (Origanum vulgare) como fotobiótico en conejos de ceba. Cuban J Agr Sci 45: 159-161.

3. Bruerton K. 2002. Antibiotic growth promoters - are there alternatives? In: Poultry Information Exchange. Queensland, Australia

4. Burt SA, Reinders RD. 2003. Antibacterial activity of selected plant essential oils against Escherichia coli O157:H7. Lett Appl Microbiol 36: 162167. doi: 10.1046/j.1472-765X.2003.01285.x

5. Castillo W, Alves M. 2007. Alimentación de lechones destetados precozmente $\mathrm{y}$ efectos en el subsecuente desempeño en el acabado. En: XX Reunión ALPA. Cusco: Asociación Latinoamericana de Producción Animal.

6. CKM. 2010. Ficha técnica de Orevitol ${ }^{\circledR}$ L. FT/OVL-02 3 10042010. [Internet]. Disponible en: http://www.ckmperu.com/ wp-content/uploads/2018/01/OrevitolL_Ficha-Tecnica.pdf 
7. Danura S. 2010. Requerimientos nutricionales y plan de alimentación para lechones. [Internet], Disponible en: http:/ /www.universoporcino.com/articulos/ nutricion_porcina_04-2010_requerimientos_nutrici-onales_y_plan de_alimentacion_para_lechones.html

8. Garcia V, Catala-Gregori P, Hernandez F, Megias MD, Madrid J. 2007. Effect of formic acid and plant extracts on growth, nutrient digestibility, intestine mucosa morphology, and meat yield of broilers. J Appl Poultry Res 16: 555-562.

9. Guerra A, Galán O, Méndez, A, Murillo A. 2008. Evaluación del efecto del extracto de orégano (Origanum vulgare) sobre algunos parámetros productivos de cerdos destetados. Tumbaga 3: 16-29.

10. Lara PE, Ortiz I, Fabian M, Aguilar $U$, Sangines JR. 2010. Harinas de hojas de plantas aromáticas como fitoterapéuticos en pollos de engorda. Pesqui Agropecu Bras 45: 294-298.

11. Lee KW, Everts H, Kappert HJ, Yeom KH, Beynen $A C$. 2003. Dietary carvacrol lowers body weight gain but improves feed conversion in female broiler chickens. J Appl Poultry Res. 12: 394-399. doi: 10.1093/japr/12.4.394

12. Martínez D. 2012. Evaluación de un producto a base de aceite esencial de orégano sobre la integridad intestinal, la capacidad de absorción de nutrientes y el comportamiento productivo de pollos de carne. Tesis de Maestría. Lima: Univ. Nacional Agraria La Molina. 364 p.

13. [MINAGRI] Ministerio Nacional de Agricultura y Riego. 2013. Anuario estadístico. Producción pecuaria e industria avícola 2013. [Internet]. Disponible en: http://siea.minagri.gob.pe/siea/ $? \mathrm{q}=$ produccion-pecuaria-e-industriaavicola

14. [MINAGRI] Ministerio Nacional de Agricultura y Riego. 2018. Al 2021 se espera un incrementar a 10 kilos el consumo per cápita de carne de cerdo. [Internet]. Disponible en: https:// www.minagri.gob.pe/portal/publicaciones-y-prensa/noticias-2018/21413-al2021-se-espera-incrementar-a-10-kilosel-consumo-per-capita-de-carne-cerdo

15. Paramio T, Manteca $X$, Milan J, Piedrafita J, Izquierdo D, Gasa J, Mateu E, Pares R. 2012. Manejo y producción de porcinos. Breve manual de aproximación a la empresa porcina para estudiantes de veterinaria. España: Univ. Autónoma de Barcelona. $52 \mathrm{p}$.

16. Parrado M, Chamorro J, Serrano L. 2006. Estudio preliminar: orégano como promotor del crecimiento en lechones destetados. Rev Med Vet 12: 81-88.

17. Pearce KL, Rosenvold K, Andersen HJ, Hopkins DL. 2011. Water distribution and mobility in meat during the conversion of muscle and ageing and the impacts on fresh meat quality attributes. A review. Meat Sci 89: 111124. doi: 10.1016/j.meatsci.2011.04.007

18. Piva G, Rossi F. 1999. Future prospects for the non-therapeutic use of antibiotics. En: XII Proceedings of the ASPA. Piacenza: Associazione per la Scienza e le Produzioni Animali.

19. Shiva C. 2007. Estudio de la actividad antimicrobiana de extractos naturales y ácidos orgánicos. Posible alternativa a los antibióticos promotores de crecimiento. Tesis doctoral. Barcelona: Univ. Autónoma de Barcelona. $173 \mathrm{p}$.

20. Shiva C, Bernal S, Sauvain M, Caldas J, Kalinowski J, Falcón N, Rojas $\boldsymbol{R}$. 2012. Evaluación del aceite esencial de oregano (Origanum vulgare) y extracto deshidratado de jengibre (Zingiber officinale) como potenciales promotores de crecimiento en pollos de engorde. Rev Inv Vet Perú 23: 160-170. doi: 10.15381/rivep.v23i2.896

21. Windisch W, Schdler K, Plitzner C, Kroismayr A. 2008. Use of phytogenic as feed additives for swine and poultry. J Anim Sci. 86: 140-148. doi: 10.2527/ jas.2007-0459 